\title{
Encapsulación de hierro: Otra estrategia para la prevención o tratamiento de la anemia por deficiencia de hierro
}

\author{
Iron encapsulation: Another strategy for the prevention or \\ treatment of iron deficiency anemia
}

\begin{abstract}
Iron deficiency anemia affects one third of the world population, causing severe health problems. Prevention and treatment strategies are based on oral supplementation and fortification of foods, but the effectiveness of these strategies has not been as expected. One explanation is that the iron compounds used have low bioavailability and produce undesired effects. Therefore, new technologies that could avoid these problems, like the encapsulation, have been explored. This technology improves the bioavailability of iron, reduces organoleptic alterations of fortified products, and decreases gastrointestinal disorders related to supplement use. These encapsulated iron products have been studied in vitro and also applied in in vivo studies to determine their efficacy against iron deficiency anemia, with promising results. Therefore, the objective of this review was to gather information about current trends in iron encapsulation as a tool to prevent or treat iron deficiency anemia.
\end{abstract}

Keywords: Encapsulation; iron; iron deficiency; anemia.

\section{INTRODUCCIÓN}

El hierro es un micromineral necesario para una amplia variedad de funciones como el transporte de oxígeno, la proliferación celular, inmunidad, síntesis de DNA, producción de energía, entre otras. La dieta humana contiene dos formas de hierro: hierro hemo y no hemo. El primero deriva de alimentos cárnicos, vísceras y sangre que contengan mioglobina y hemoglobina. El hierro no hemo, que se encuentra en cereales, lácteos, legumbres y vegetales, está presente en los alimentos como sal férrica $\left(\mathrm{Fe}^{+3}\right)$ o ferrosa $\left(\mathrm{Fe}^{+2}\right)$. El cuerpo de un adulto contiene 3-4 $\mathrm{g}$ de hierro, que se obtiene desde los alimentos y lo normal en la dieta occidental es que contenga $7 \mathrm{mg}$ de hierro por $1.000 \mathrm{kcal}$; sin embargo, sólo 1-2 mg normalmente son absorbidos cada día'.

El hierro es un micromineral cuya homeostasis está controlada principalmente por la absorción de hierro

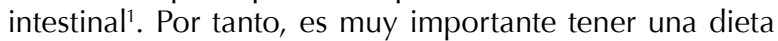

\author{
Emerson Durán ${ }^{1}$, Claudia Villalobos ${ }^{1}$, Osmaly Churio ${ }^{1}$,
} Fernando Pizarro², Carolina Valenzuela ${ }^{1}$.

1. Departamento de Fomento de la Producción Animal, Facultad de Ciencias Veterinarias y Pecuarias, Universidad de Chile. Avda. Santa Rosa 11.735, La Pintana, Santiago, Chile. 2. Laboratorio de Micronutrientes, Instituto de Nutrición y Tecnología de los Alimentos, Universidad de Chile. Av. El Líbano 5524 Macul, Santiago de Chile.

Dirigir correspondencia a: Carolina Valenzuela V. Departamento de Fomento de la Producción Animal, Facultad de Ciencias Veterinarias y Pecuarias, Universidad de Chile. Avda. Santa Rosa 11.735, La Pintana. Dirección postal: Casilla 2, correo 15, La Granja. Santiago, Chile. Teléfono: 229785676. Email: cvalenzuelav@u.uchile.cl

Este trabajo fue recibido el 11 de enero de 2017. Aceptado con modificaciones el 19 de mayo de 2017 Aceptado para ser publicado el 14 de junio de 2017.

balanceada en cuanto al aporte de hierro, sino se presenta su deficiencia que conduce a anemia. Una carencia que afecta a un tercio de la población mundial, principalmente de países en vías de desarrollo. La anemia compromete la salud de mujeres en edad fértil, embarazadas, niños preescolares y adultos mayores. Entre sus efectos negativos para la salud se reconocen: retraso del crecimiento, problemas de desarrollo cognitivo, bajo rendimiento escolar, disminución de la productividad, entre otras afecciones ${ }^{2}$. Una de las causas de este tipo de anemia son el consumo de dietas bajas en hierro y el desbalance entre el consumo de dietas altas en hierro no hemo (tres cuartas partes del hierro de la dieta), que presenta una baja biodisponibilidad, respecto al hierro hemo ${ }^{3}$. Otras causas son el síndrome de mala absorción, insuficiencia renal, malaria, entre otras.

Como estrategias de prevención y/o tratamiento de la anemia, la suplementación oral y la fortificación de alimentos con hierro son los más usados, los cuales a pesar de estar 
bastante masificados, no han tenido el efecto esperado en la disminución de la prevalencia de la anemia y presentan ciertas desventajas que hacen que los beneficiarios no realicen la terapia completa y/o dejen de utilizar el alimento fortificado, nombrando entre las principales: 1) cambios negativos en las características organolépticas de los alimentos fortificados con hierro, 2) malestares gastrointestinales, 3) efecto reducido en aumentar niveles de hemoglobina debido a que se usa generalmente hierro no hemo que tiene baja biodisponibilidad, o 4) simplemente olvido del consumo de suplementos y alimentos fortificados ${ }^{4-7}$.

Los primeros de estos motivos han sido mejorados utilizando la tecnología de encapsulación de hierro que es un proceso de micro-empaquetamiento en donde una gran variedad de compuestos de hierro han sido protegidos con diversos materiales muralla (matriz) produciendo partículas de varios tamaños (nanómetros a milímetros) mediante diversas metodologías ${ }^{8}$. Así se han generado diferentes formas de hierro encapsuladas, a las cuales se ha probado su efectividad in vitro, pero también in vivo con resultados prometedores para reducir la anemia por deficiencia de hierro. Por tanto, el objetivo de esta revisión fue recopilar información acerca de las tendencias actuales de encapsulación de hierro como una herramienta para prevenir o tratar la anemia por deficiencia de hierro.

\section{DESARROLLO}

Manejo de la anemia por deficiencia de hierro

La anemia genera grandes pérdidas económicas, difíciles de cuantificar ${ }^{9}$, y aunque en materia de salud pública se han realizado varios esfuerzos para su prevención, los resultados no han sido los esperados ${ }^{2,10}$. Actualmente, las principales estrategias de prevención de la anemia han sido la suplementación oral y la fortificación de alimentos. Los suplementos más usados se basan principalmente en mezclas farmacéuticas de diferentes formas de hierro como: fumarato ferroso, sulfato ferroso, gluconato ferroso, hierro aminoquelado, etc., que pueden estar combinados con otros compuestos como ácido ascórbico que promueven la absorción de hierro no hemo, vitamina B12, ácido fólico, entre los principales.

El grupo etario de más alto riesgo de padecer de anemia por deficiencia de hierro son las embarazadas. Para países como el nuestro donde las mujeres en edad fértil presentan una prevalencia moderada de anemia, se recomienda suplementar a todas las embarazadas a partir del segundo trimestre de la gestación con una dosis diaria de $60 \mathrm{mg}$ de sulfato ferroso ${ }^{11}$. Se describe que los suplementos orales de hierro son deseables como primera línea de terapia porque son seguros, de bajo costo y efectivos mejorando el estado de hierro corporal. Sin embargo, esta terapia puede ser limitada por su controversial impacto debido a la baja biodisponibilidad de algunos suplementos y a sus efectos secundarios intestinales ${ }^{12}$. Para reducir estos efectos se han desarrollado suplementos de hierro con recubrimiento entérico y de liberación controlada, los cuales están asociados con menores efectos secundarios. Sin embargo, de todas formas hay un alto porcentaje de personas que suspende u olvida la terapia de suplementación ${ }^{5}$.

Otra forma para mejorar el estado de nutrición de hierro es la fortificación de alimentos que sean altamente consumidos por la población, con el fin de incrementar la ingesta del nutriente carente. Para éste fin los compuestos de hierro que son comúnmente usados son: sulfato ferroso, gluconato ferroso, lactato ferroso, citrato de amonio férrico, fumarato ferroso, succinato ferroso, sacarato férrico, hierro EDTA, hemoglobina y eritrocitos ${ }^{13,14}$. Entre los alimentos fortificados con hierro se mencionan: cereales, sal, azúcar, leche, condimentos y café13-16. A pesar de su potencial, la fortificación de alimentos tiene como principal limitante los cambios indeseables de color y sabor en los alimentos debido a los efectos oxidantes del hierro ${ }^{6,7}$.

\section{Razones para encapsular hierro}

Los motivos que han llevado a encapsular este micronutriente son diversos:

1) Protección de los compuestos de hierro de factores ambientales adversos y de su paso por el tracto gastrointestinal, y así mejorar la biodisponibilidad del hierro no hemo, ya que existen varios factores intraluminales que disminuyen su absorción (fibra, taninos, polifenoles, otros minerales como $\mathrm{Ca}, \mathrm{Cu}$ y Zn, entre otros) (Figura 1A) ${ }^{1}$.

2) Enmascarar el sabor metálico que presenta el hierro (no hemo y hemo) cuando es incorporado en alimentos ${ }^{13}$, ya que los materiales encapsulantes al cumplir una función de barrera previenen el contacto directo entre el hierro y los receptores gustativos. Incluso en materiales núcleos de muy mal sabor como el hierro, se puede proveer una doble o múltiple encapsulación con diferentes materiales encapsulantes que pueden incluir en su formulación agentes saborizantes (Figura 1B).

3) Disminuir los cambios de color que genera el hierro cuando es incorporado en alimentos (Figura 1C). Muchos de los compuestos de hierro usados en fortificación tienen coloraciones marrones oscuras (fumarato ferroso, eritrocitos atomizados, bis-glicinato ferroso, entre otros), que alteran al alimento original. Como fue el caso de leche fortificada con hierro, en donde la leche que no contenía hierro encapsulado mostró alteraciones de color y sabor, percibidas en un análisis sensorial ${ }^{17}$. Además, de que el hierro causa también reacciones de decoloración, por ejemplo cereales que se han vuelto grises o verdes al adherirles sulfato ferroso ${ }^{18}$.

4) Reducir el efecto catalizador del hierro sobre la oxidación de grasas, ya que reduce la vida útil del producto $^{19}$.

5) Disminuir los efectos adversos gastrointestinales (Figura 1C), ya que las sales de hierro solubles pueden producir varios efectos secundarios tales como: oscurecimiento dental, dolor abdominal, pirosis, náuseas, diarrea, estreñimiento y heces negras ${ }^{20}$. 
6) Disminuir la precipitación de hierro en estómago. Para esto se deben seleccionar ciertos tipos de materiales encapsulantes para formar micro-partículas de hierro que liberen el mínimo contenido en estómago, por ejemplo el alginato de sodio que soporta las condiciones ácidas del estómago, y que este material se degrade lentamente en el duodeno, lugar en donde se absorbe el hierro. Esto se conoce con el nombre de liberación controlada (Figura 1D).

7) La encapsulación también permite la posibilidad de mezclar en una misma micro-partícula/micro-cápsula/ liposoma, dos fuentes diferentes de hierro (Figura 1A), ya que el hierro no hemo se absorbe por el transportador de metales divalentes 1 (DMT1), y el hemo mediante la proteína transportadora del grupo hemo 1 (HCP1), y ambos receptores son saturables, ${ }^{1,21}$. También con compuestos que promuevan su absorción. Es el caso de la encapsulación mixta de hierro hemo/no hemo ${ }^{22,23}$, ya que el hierro hemo promueve la absorción de hierro no hemo ${ }^{24}$, ácido ascórbico (Figura 1A) que aumenta la absorción de hierro no hemo ${ }^{25}$, o simplemente entregar dosis de varios micronutrientes esenciales como es el caso de las chispitas o "sprinkles"26.

Métodos para encapsular hierro y obtención de diferentes compuestos de hierro encapsulado

Existe una gran diversidad de métodos de encapsulación de hierro aplicables a la industria de los alimentos como el secado por aspersión (atomización), entrampamiento en liposomas y gelación iónica, entre los principales ${ }^{27}$.

El secado por aspersión implica la pulverización de una solución que contiene hierro como material núcleo y algún material encapsulante como material muralla (carbohidratos como maltodextrina, derivados del almidón, celulosa, alginato, quitosano y proteínas derivadas de suero de leche, soya, otras $)^{28}$. Generalmente se elabora una dispersión de baja o media viscosidad, en donde el material núcleo se suspende en diferentes proporciones en soluciones acuosas del material encapsulante, la cual posteriormente alimenta al equipo de atomización ("spray dried"), que deshidrata estas dispersiones, obteniendo un material en formato polvo formado por millones de micro-partículas cuyo tamaño puede variar de 10 a $400 \mu \mathrm{m}^{29,30}$. Este método de encapsulación es uno de los más utilizados en la industria de alimentos debido a sus altos rendimientos, bajos costos y ventajas de almacenamiento. Sin embargo, no es el más usado para la encapsulación de hierro debido a que los materiales encapsulantes que permite usar el equipo de atomización convencional son generalmente solubles en agua, y no proporcionan una protección suficiente frente a la oxidación del hierro y a sus propiedades organolépticas adversas, sobre todo cuando se piensa en el uso de hierro encapsulado para la fortificación de alimentos líquidos o suplementación oral ${ }^{29,30}$.

El entrampamiento en liposomas consiste en elaborar emulsiones $(\mathrm{o} / \mathrm{w})$ y/o vesículas compuestas de lípidos
A
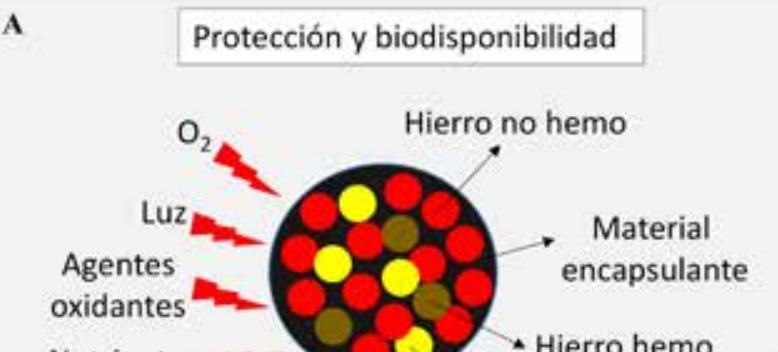
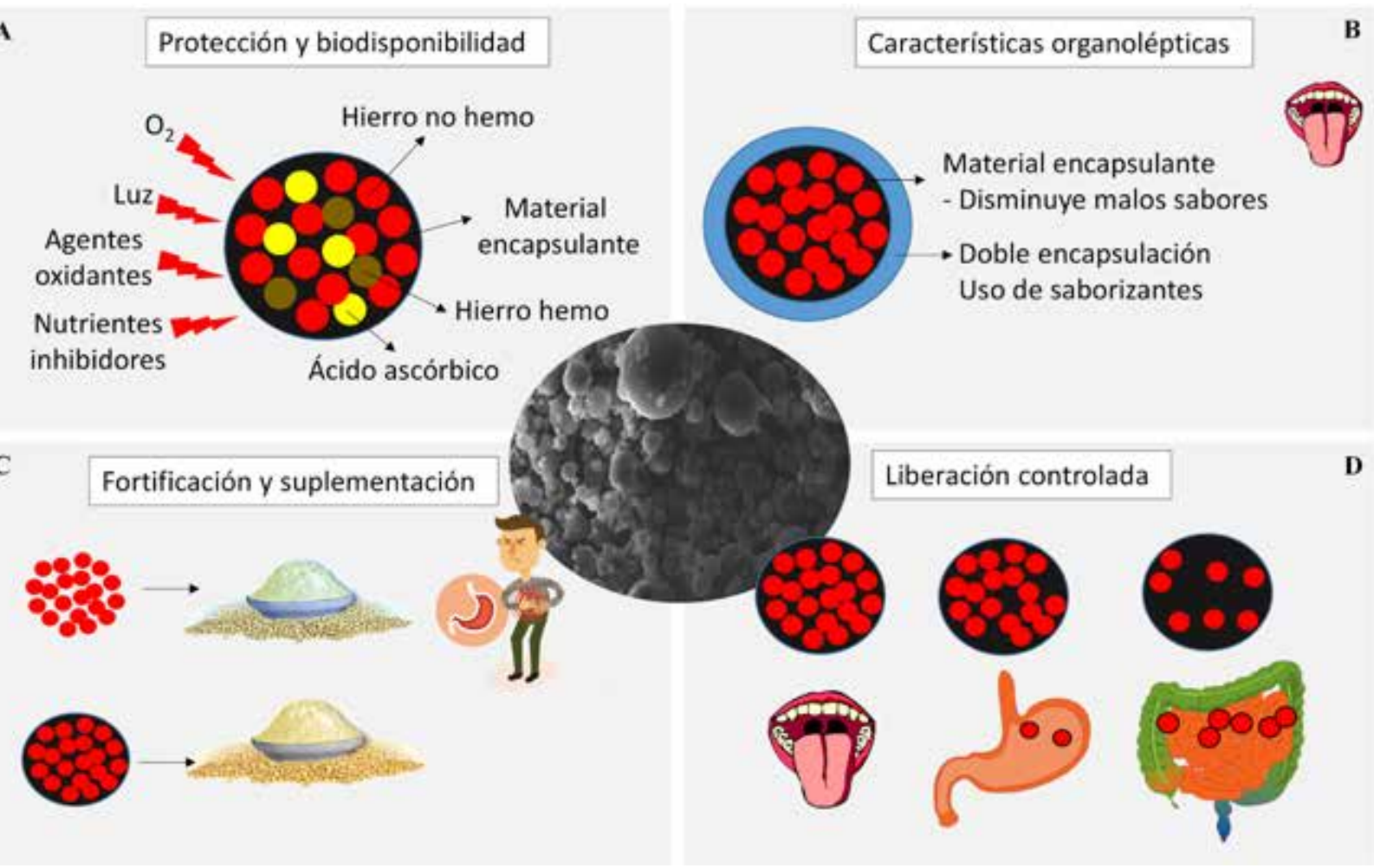
(mezclas de fosfolípidos, lecitinas, colesterol, polisorbatos) que encapsulan el hierro por varios métodos, sin embargo el más aplicado es la rota-evaporación. Este método consiste en elaborar mezclas de lípidos capaces de formar una lámina o película fina lipídica después de ser disueltos en solventes orgánicos (Ej: cloroformo, metanol) y sometidos a rotaevaporación para eliminar estos solventes. Posteriormente la película lipídica debe ser hidratada con un una solución acuosa de características hidrofílicas que contenga el material núcleo que se quiere encapsular. Así es posible obtener una suspensión homogénea primaria multilamelar de liposomas. Para reducir el tamaño de los liposomas, se pueden utilizar equipos como baño ultrasónico. Con esta técnica se pueden obtener liposomas de tamaños variables entre 0,2 a $5.000 \mu \mathrm{m}^{30,31}$.

El uso de esta técnica es limitado debido a la inestabilidad física y química de los liposomas, bajos rendimientos y eficiencia de encapsulación, liberación prematura del hierro desde los liposomas, y altos costos en su procesamiento. Teóricamente la estabilidad del hierro en este método debe ser alta, siempre que los liposomas se mantengan estructuralmente intactos ${ }^{27}$. Lo que es difícil de obtener si se aplican como parte de un suplemento oral ya que el sólo paso por el tracto gastrointestinal los desestabilizaría, debido a la presencia de lipasa pancreática y ácidos biliares en el intestino delgado, que acelera su desintegración.

La gelación iónica consiste en la reacción química entre ciertos polisacáridos, como el alginato de sodio y una solución de cationes divalentes (Ilamada solución reticulante o gelificante), siendo el más usado el $\mathrm{CaCl}^{2}$, que reaccionan formando estructuras de "cajas de huevos" en donde quedan atrapados los compuestos que se pretenden encapsular. Para encapsular hierro mediante esta técnica en necesario preparar una solución de alginato de sodio en concentraciones de $1,5-3 \% \mathrm{p} / \mathrm{v}$ en la cual diferentes fuentes de hierro pueden ser dispersadas, luego con la ayuda de jeringas, pipetas, aspersores o disco atomizador, se gotean las dispersiones en la solución gelificante formando perlas de distintos tamaños dependiendo del instrumento usado, desde 200 a $5.000 \mu \mathrm{m}^{30}$. Con esta técnica se ha logrado encapsular eficientemente hierro hemo proveniente de eritrocitos bovinos ${ }^{32,33}$ y también diferentes tipos de hierro no hemo ${ }^{34}$.

También es posible agregar el hierro en la solución gelificante, cuando las sales de hierro presentan incompatibilidad electrolítica para formar perlas por el método convencional ${ }^{34}$. Este método de encapsulación es bastante prometedor para suplementación de hierro oral, porque el alginato es un material que libera una baja concentración del hierro encapsulado a nivel gástrico, y a nivel intestinal la liberación es controlada en el tiempo, y se produce una liberación casi completa después de 3 horas de digestión en condiciones in vitro ${ }^{33}$.

Los principales desafíos en la encapsulación de hierro son la estabilidad del producto encapsulado y los $\operatorname{costos}^{29}$. En el caso de los productos líquidos la estabilidad puede ser un problema mayor, ya que tras el almacenamiento, las partículas de hierro encapsulado pueden sedimentar en productos como la leche y jugos de frutas ${ }^{27}$. Para determinar que método y materiales de encapsulación son los más efectivos a utilizar se debe tener claridad del producto de hierro encapsulado que se quiere generar, en qué tipo de programa se va a utilizar como fortificación de alimentos o suplementación, el público objetivo, las condiciones de vehiculización y almacenamiento, entre las principales.

En la Figura 2 se muestra el aspecto y morfología de algunos de los compuestos de hierro encapsulados. En la Figura $2 \mathrm{~A}$, B y C se presentan perlas, en donde diferentes tipos de hierro fueron encapsulados por gelación iónica, utilizando como material encapsulante alginato de sodio ${ }^{33,35}$. Estas perlas son de tamaño grande, entre 1 a $2 \mathrm{~mm}^{31-36}$. En la Figura $2 \mathrm{D}$ y E se observan liposomas. Este método para encapsular hierro se caracteriza por obtener vesículas lipídicas de tamaño nanométrico que son observadas mediante microscopía electrónica de trasmisión. En la Figura $2 \mathrm{~F}$ se observa el hierro encapsulado como una emulsión, en donde el tamaño de las gotas puede variar ${ }^{37}$, pero mediante el uso de técnicas como baño ultrasónico o sonicación se puede disminuir el tamaño a micras o nanómetros. En la Figura 2 G-I se presentan diferentes micropartículas elaboradas con distintos compuestos de hierro observadas mediante microscopía electrónica de barrido $^{38,39}$. Los tamaños de éstas también son variables (generalmente en micras). Los métodos para obtener éstas micropartículas son muy variados, observando que para el secado por atomización se pueden obtener formas esféricas (Figura $2 \mathrm{H}$ ), o con mayores irregularidades y concavidades en su superficie (Figura $2 \mathrm{G}$ ).

Aplicaciones de hierro encapsulado en nutrición

Las publicaciones relacionadas con la encapsulación de hierro y su estudio a nivel de laboratorio en diferentes investigaciones in vitro se muestran en la Tabla $1^{31-33,40-45}$. Las principales características que se estudian son: el contenido de hierro, el aspecto y la morfología de las micropartículas/ liposomas/perlas, tamaño, potencial Z, eficiencia de encapsulación, rendimiento, estabilidad del producto al almacenamiento o en una matriz alimenticia como el caso de los alimentos fortificados, efecto sobre la oxidación, y características organolépticas. El principal compuesto de hierro utilizado para la encapsulación es el sulfato ferroso, que es considerado como "gold standard" y hay pocos estudios donde se encapsula hierro hemo. Los métodos de encapsulación usados principalmente son entrampamiento en liposomas, extrusión y gelación iónica. De estos los que presentan una mejor eficiencia de encapsulación son la extrusión y la gelación iónica. Particularmente se observó un bajo porcentaje de liberación de hierro desde perlas de alginato a nivel gástrico y alto porcentaje a nivel intestinal, lo que hace adecuado este sistema para suplementación oral de hierro. Las principales conclusiones de estos estudios son que el hierro es un compuesto fácil de encapsular y 


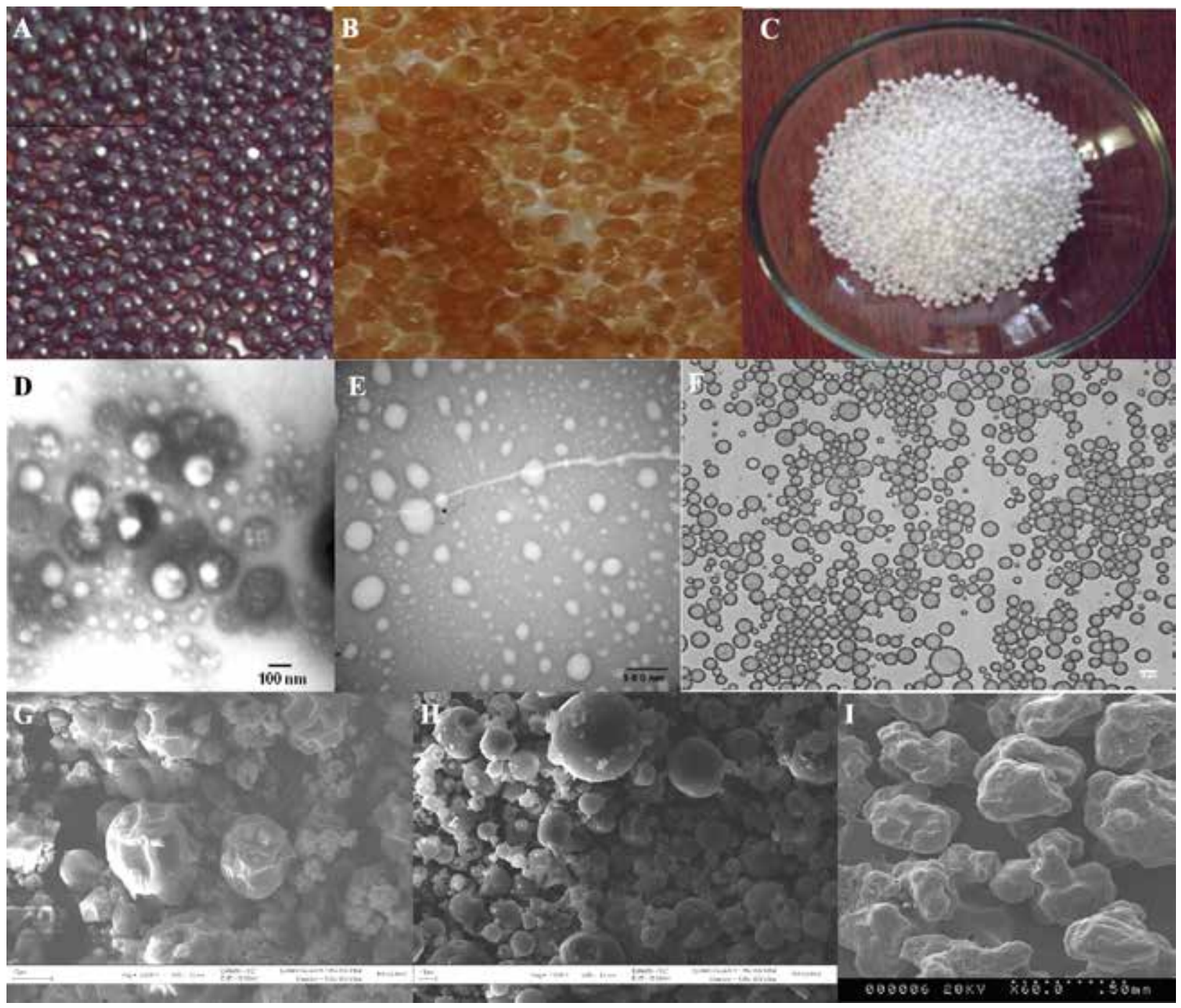

que hay varias técnicas para medir la efectividad de la encapsulación, basados principalmente en digestiones in vitro simulando condiciones gastrointestinales y modelos celulares como Caco-2. El hierro encapsulado disminuye notoriamente los cambios organolépticos adversos que genera cuando es añadido en alimentos fortificados y disminuye los procesos de oxidación de grasas.

En relación a los estudios in vivo de productos con hierro encapsulado, algunas de las investigaciones relevantes se presentan en la Tabla $2^{38,46-52}$. Estos productos se han probado en modelos animales como rata y cerdo, como también en humanos. Generalmente se observa que el principal material usado para la encapsulación es nuevamente el sulfato ferroso, y que el método de encapsulación más usado es el entrampamiento en liposomas. En varios estudios se ha demostrado que efectivamente la encapsulación de hierro mejora su biodisponibilidad, sin embargo, en algunas investigaciones no se observó un efecto de la encapsulación cuando se comparó el hierro encapsulado con las mismas formas sin encapsular.

En conclusión, se observa que el hierro encapsulado aumenta la eficiencia de suplementación de este en comparación al no encapsulado, por medio del aumento de su biodisponibilidad en el organismo y disminución de sus características organolépticas negativas. Teniendo un claro impacto en los índices hematológicos revisados en los diversos estudios, por lo que tiene un potencial en la fortificación de alimentos y la suplementación oral y parenteral.

Productos con hierro fortificado existentes en el mercado Actualmente en el mercado existen productos comerciales que usan la tecnología de encapsulación de hierro, uno de ellos son los "sprinkles". Estos son sobres individuales que 


\section{Tabla 1}

Aplicaciones in vitro de hierro encapsulado.

\section{Autor Compuesto encapsulado}

[40] Sal férrica sódica de EDTA, fuma-rato ferroso, piro-fosfato férrico y SunActive $®$

[37] $\mathrm{Fe}^{3+}$

[41] Fumarato ferroso

[42] Sulfato ferroso $y$ sulfato de amonio férrico

Glicinato ferroso

Sulfato ferroso

Sulfato ferroso

Hierro hemínico

Gelación iónica

Emulsión y solvente

Evaporación del solvente

Gelación iónica

Entrampamiento en liposoma y aspersión por enfriamiento evaporación del

Aplicación

Se buscó desarrollar una formulación óptima de Ultra Rice ${ }^{\circledR}$ (un tipo de arroz fortificado con hierro, vitaminas y zinc). Para esto se probaron 4 formas de hierro diferentes. Las formas más biodisponibles fueron hierro-Na-EDTA y fumarato ferroso, pero causaron mayor pérdida de tiamina, rancidez y color oscuro del arroz. El pirofosfato férrico y SunActive ${ }^{\circledR}$ mostraron mayor estabilidad y mejores propiedades sensoriales, pero menor solubilidad.

El hierro fue eficientemente encapsulado en la fase interna de emulsiones W/O/W con aceite de pescado y de maíz. Sin embargo, se observó que las emulsiones que contenían hierro encapsulado promovían la oxidación de lípidos al añadirse a emulsiones de aceite de pescado en contraste a las que no se les añadió hierro.

Se elaboró una pre-mezcla de hierro encapsulado biodisponible y estable, gracias al proceso de extrusión, llegando a una consistencia adecuada para su incorporación en la sal yodada.

Se encapsuló hierro con 2 métodos distintos basados en recubrimientos lipídicos diferentes. Los microencapsulados obtenidos se añadieron en leche y se observaron mejores propiedades sensoriales con la aspersión por enfriamiento usando ésteres de ácidos grasos.

Se lograron formar nanoliposomas con alta eficiencia de encapsulación y estables en condiciones gastrointestinales in vitro durante 5 horas, los cuales pueden ser adecuados para su uso en administración oral de glicinato ferroso. La posibilidad de dirigir el glicinato ferroso a tejidos específicos será altamente influenciada por el tamaño del nanoliposoma, las propiedades superficiales y la estabilidad de estos.

Emulsión/Gelación Se fortificó un yogurt con hierro encapsulado. No se afectó el crecimiento de las bacterias ácido lácticas. Se observó una reducción de la oxidación en el yogurt con hierro encapsulado comparado al sin encapsular.

Se observó una mayor absorción del hierro encapsulado comparado con el sin encapsular en modelo de células Caco-2. Se demostró que el contenido inicial de hierro y la carga superficial tienen una alta influencia en las características fisicoquímicas de absorción del hierro in vitro. La adición de quitosano en una concentración óptima afectó positivamente la absorción del hierro.

Se logró encapsular hierro hemínico (eritrocitos bovinos atomizados), eficientemente en perlas de alginato de sodio, pero la concentración de hierro de las perlas fue baja.

Se prepararon microcápsulas con una mezcla de goma arábica, maltodextrina y almidón modificado. Las microcápsulas tuvieron elevada estabilidad y eficiencia de encapsulación, al agregarlas a la leche se obtuvo una buena biodisponibilidad del hierro y una baja oxidación.

Se observó un bajo porcentaje de liberación de hierro desde perlas de alginato a nivel gástrico y alto porcentaje a nivel intestinal. Lo que hace adecuado este sistema para suplementación oral. 
Tabla 2

Aplicaciones in vivo de hierro encapsulado.

\section{Autor Compuesto encapsulado}

[46] Fumarato ferroso

[47] Sulfato ferroso

[48] Fumarato ferroso

[49] Sulfato de hierro

[50] Sacarato férrico y sulfato ferroso

[51] Hemina y citrato férrico

[52] Citrato de amonio férrico

[38] Eritrocitos bovinos atomizados y sulfato ferroso

\section{Método de encapsulación}

Entrampamiento en liposomas

Entrampamiento en liposomas

Entrampamiento en liposomas

Secado por atomización

No especificado. Producto comercial de AB-Fortis ${ }^{\circledR}$

Entrampamiento en liposomas

Entrampamiento en liposomas

Secado por atomización

\section{Aplicación}

El uso de "sprinkles" (hierro más otros micronutrientes encapsulados) en un grupo de niños de 6 a 18 meses con anemia por deficiencia de hierro mejoró su estado de nutrición de hierro, pero de manera similar al grupo control (gotas de sulfato ferroso).

Se fortificó sal yodada con hierro encapsulado que se usó en la preparación de comidas de niños de 6 a 15 años, disminuyendo la anemia por deficiencia de hierro desde un $35 \%$ a un $8 \%$.

El uso de "sprinkles" en papillas en base a maíz entregadas a niños con distintos estados de nutrición de hierro mejoró el estado hematológico de los niños, observándose un mayor efecto en los anémicos. No se observó un efecto de la dosis de sprinkles utilizada.

Se le ofrecieron rollos con harina de trigo fortificada con hierro microencapsulado, a niños de entre 2 y 6 años. Se observó que fueron bien tolerados y aumentaron los niveles de hemoglobina y disminuyeron los índices de anemia por deficiencia de hierro.

Se fortificó leche con $A B$-Fortis ${ }^{\circledR}$ y sulfato ferroso y se comparó su absorción. No hubo diferencias significativas en los parámetros sanguíneos entre ambos grupos en humanos adultos con un estado de nutrición de hierro normal.

La aplicación intraperitoneal de citrato ferrico y hierro hemo encapsulado en ratas con anemia por inflamación, aumentaron en $119 \%$ y $54 \%$ los niveles de hierro sérico comparados con éstas mismas formas de hierro sin encapsular. También los liposomas de hierro hemo regularon la hepcidina.

Se le administró hierro encapsulado a ratas con anemia por deficiencia de hierro en ejercicio extenuante. La suplementación con hierro encapsulado revirtió la anemia, y puede ser usado potencialmente para tratar la anemia asociada al ejercicio en humanos.

Fue posible prevenir la anemia por deficiencia de hierro típica del cerdo lactante, al destete, con el uso de micropartículas de mezcla entre hierro hemo/no hemo que se entregaron como suplemento oral a cerdos lactantes. El efecto hematológico de 3 dosis de este suplemento fue similar al tratamiento parenteral con hierro dextrano usado rutinariamente en los criaderos de cerdo. contienen una mezcla de micronutrientes (hierro, zinc, ácido fólico y vitaminas $\mathrm{A}$ y $\mathrm{C}$ ), encapsulados con lípidos, que se espolvorean sobre un alimento (por ejemplo papillas de cereales) ${ }^{26}$. Los "sprinkles" fueron diseñados para reducir la prevalencia de anemia en los niños. Según Zlotkin et al. ${ }^{53,54}$ quienes son los creadores de esta estrategia, el uso de "sprinkles" en bebés y niños anémicos provenientes de África mejora la absorción de hierro y reduce la deficiencia de hierro. Sin embargo, esta mejora no siempre fue superior a los resultados obtenidos cuando se usaron los métodos comunes de suplementación/fortificación de hierro, pero sí el uso de "sprinkles" fue mejor aceptado y tolerado por los niños que el uso de gotas de sulfato ferroso.

Otro producto es el $A B$-fortis ${ }^{\circledR}$ que es una fórmula patentada basada en un sistema de micro-encapsulación de hierro (sacarato férrico) por gelación iónica. Según datos 
de la empresa que lo comercializa (Frutarom http://www. frutaromhealth.com/ab-fortis/), este producto es un polvo café, que contiene $40 \%$ de hierro, la dosis a usar en una comida típica es entre 6 a 35 mg, y que su utilización evita el desagradable sabor metálico y los efectos secundarios clásicos que suelen tener los suplementos de hierro. También que es estable a altas temperaturas, por lo que puede ser incorporado en una amplia gama de alimentos y comidas. Sin embargo, según los resultados del estudio realizado por Contreras et al. ${ }^{50}$, la absorción entre el sacarato férrico sin encapsular y $A B$-fortis ${ }^{\circledR}$ no difieren significativamente.

La principal proyección de la técnica de encapsulación para hierro, es su aplicación en la industria alimentaria para mejorar algunas características de los variados alimentos que están fortificados con hierro. En los cuales se podría mejorar la biodisponibilidad del hierro, ya que las formas de hierro más comúnmente utilizadas son sales ferrosas o férricas de baja biodisponibilidad; y reducir algunas características adversas que el hierro confiere a los alimentos. Así obtener alimentos fortificados con una mejor respuesta farmacéutica en relación a la mejora del estado de nutrición de hierro de los consumidores. Entre los principales alimentos fortificados se mencionan: lácteos (queso crema, cheddar, mozzarella, cottage, yogurt, leche con chocolate) ${ }^{55}$, productos de panadería (pan, galletas, cereales para el desayuno), y cereales (harina de maíz, harina de trigo, papillas de cereales para bebés, arroz) ${ }^{56}$.

\section{CONCLUSIONES}

El hierro es un micromineral fácil de encapsular, ya que esta técnica se puede realizar con varios compuestos de hierro y mediante diversas metodologías. La encapsulación de hierro es un método efectivo para mejorar la biodisponibilidad del hierro, pero principalmente reducir las características organolépticas adversas en alimentos fortificados. Gracias a las ventajas de esta tecnología es posible formular suplementos de hierro que sean mejor tolerados y productos alimenticios fortificados con compuestos de hierro que sean habitualmente consumidos en las distintas poblaciones en riesgo. De esta forma la tecnología de encapsulación puede contribuir en el desafío de disminuir la alta prevalencia de anemia por deficiencia de hierro.

\section{RESUMEN}

La anemia por deficiencia de hierro afecta a un tercio de la población mundial, causando severos problemas de salud. Las estrategias de prevención y tratamiento se basan en la suplementación oral y fortificación de alimentos, pero su eficacia no ha sido la esperada. Una de las causas se debe a que los compuestos de hierro utilizados tienen baja biodisponibilidad y producen efectos indeseados. Por esto, se han buscado nuevas tecnologías que pudiesen evitar estos problemas, como la encapsulación. Esta tecnología mejora la biodisponibilidad del hierro, reduce alteraciones organolépticas de los productos fortificados, y disminuye los trastornos gastrointestinales del uso de suplementos, entre las principales ventajas. Estos productos de hierro encapsulados han sido estudiados in vitro, y también aplicados en estudios in vivo para determinar su eficacia contra la anemia por deficiencia de hierro, con promisorios resultados. Por tanto, el objetivo de esta revisión fue recopilar información acerca de las tendencias actuales de encapsulación de hierro como una herramienta para prevenir o tratar la anemia por deficiencia de hierro.

Palabras clave: Encapsulación; hierro, deficiencia de hierro, anemia.

\section{BIBLIOGRAFÍA}

1. Conrad M, Umbreit J. Iron absorption and transport-an update. Am J Hematol 2000; 64: 287-298.

2. Johnson-Wimbley $T$, Graham D. Diagnosis and management of iron deficiency anemia in the 21st century. Therap Adv Gastroenterol 2011; 4(3): 177-184.

3. Carpenter C, Mahoney A. Contributions of heme and nonheme iron to human nutrition. CrC Cr Rev Food Sci 1992; 31: 333-367.

4. Benito $P$, Miller D. Iron absorption and bioavailability: an updated review. Nutr Res 1998; 18: 581-603.

5. Coplin M, Schuette S, Leichtmann G, Lashner B. Tolerability of iron: a comparison of bis-glycino iron II and ferrous sulfate. Clin Ther 1991; 13: 606-612.

6. Douglas F, Rainey N, Wong N, Edmondson L, La Croix D. Color, flavor, and iron bioavailability in iron-fortified chocolate milk. I Dairy Sci 1981; 64: 1785-1793.

7. Hurrel R. How to ensure adequate iron absorption from iron-fortified food. Nutr Rev 2002; 60: 7-15.

8. Zimmermann M, Windhab E. Encapsulation of iron and other micronutrients for food fortification. In Encapsulation Technologies for Active Food Ingredients and Food Processing, New York, 2010, p. 187-209.

9. Horton $S$, Ross J. The economics of iron deficiency. Food policy 2002; 28(1): 51-75.

10. Lynch $S$. The impact of iron fortification on nutritional anaemia. Best Pract Res Cl Ha 2005; 18(2): 333-346.

11. United Nations Children's. United Nations University. World Health Organization. Iron deficiency anaemia: assessment, prevention, and control. A guide for programme managers. World Health Organization, Geneva, 2001.

12. Hallberg L, Ryttinger L, Sölvell L. Side effects of oral iron therapy a double-blind study of different iron compounds in tablet form. Acta Med Scand 1966; 180(S459): 3-10.

13. Hurrell R. Preventing iron deficiency through food fortification. Nutr Rev 1997; 55(6): 210-222.

14. Martínez-Navarrete N, Camacho M, Martínez-Lahuerta J, Martínez-Monzó J, Fito P. Iron deficiency and iron fortified foods-a review. Food Res Int 2002; 35(2): 225-231.

15. Walter $T$, Hertrampf E, Pizarro F. Effect of bovine-hemoglobinfortified cookies on iron status of schoolchildren: a nationwide program in Chile. Am J Clin Nutr 1993; 57: 190-194.

16. Huma N, Salim-Ur-Rehman, Anjum F, Murtaza M, Sheikh M. Food fortification strategy-preventing iron deficiency anemia: a review. Crit Rev Food Sci Nutr 2007; 47(3): 259-265.

17. Kwak H, Yang K, Ahn J. Microencapsulated iron for milk fortification. J Agric Food Chem 2003; 51(26): 7770-7774.

18. Hurrell, R. Bioavailability of different iron compounds used to fortify formulas and cereals: technological problems. Nestle Nutrition Workshop Series., USA, 1984. 
19. Hurrell $R$, Furniss D, Burri J. Iron fortification of infant cereals: a proposal for the use of ferrous fumarate or ferrous succinate. Am J Clin Nutr 1989; 49: 1274-1282

20. Mora J. Iron supplementation: overcoming technical and practical barriers. I Nutr 2002; 132(4): 853-855.

21. Arredondo $M$, Kloosterman J, Núñez $S$, Segovia $F$, Candia $V$, Flores $S$, Le Blanc S, Olivares $M$, Pizarro F. Heme iron uptake by caco-2 cells is a saturable temperature sensitive and modulated by extracellular $\mathrm{pH}$ and potassium. Biol Trace Elem Res 2008; 125(2): 109-119.

22. Campos M, Pallarés I, Moratalla A, López-Aliaga I, GómezAyala $A$, Hartiti $S$, Alférez $M$, Barrionuevo $M$, Lisbona $F$. Bioavailability of $\mathrm{Fe}, \mathrm{Ca}, \mathrm{P}$ and $\mathrm{Mg}$ in deficient rats treated with different sources of dietary iron. Nutr Res 1996; 16(4): 683-696.

23. Ramos M, Campos M. Bioavailability of $\mathrm{Fe}, \mathrm{Cu}, \mathrm{Zn}$ and antioxidant defence in anemic rat supplemented with a mixture of heme/non-heme Fe. J Food Nutr Res 2013; 52(2): 128-138.

24. Hooda J, Shah A, Zhang L. Heme, an essential nutrient from dietary proteins, critically impacts diverse physiological and pathological processes. Nutrients 2014; 6: 1080-1102.

25. Stekel A, Olivares $M$, Cayazzo M, Chadud P, Llaguno S, Pizarro F. Prevention of iron deficiency by milk fortification. II. A field trial with a full-fat acidified milk. Am J Clin Nutr 1988; 47: 265-269.

26. Sprinkles Global Health Initiative, "sprinkles" (chispitas nutricionales) para uso en los bebés y niños pequeños: directrices sobre las recomendaciones de uso y un programa de seguimiento y evaluación. 2010.

27. Zuidam N. An industry perspective on the advantages and disadvantages of iron micronutrient delivery systems. In Garti, N., McClements, D. J. (Eds.). Encapsulation Technologies and Delivery Systems for Food Ingredients and Nutraceuticals. Woodhead Publishing Ltd., Cambridge, UK. 2012, p. 505-540.

28. Fang Z, Bhandari B. Spray drying, freeze drying and related processes for food ingredient and nutraceutical encapsulation. In: Garti, N., McClements, D. J. (Eds.). Encapsulation Technologies and Delivery Systems for Food Ingredients and Nutraceuticals. Woodh Publ Ltd., Cambridge, UK., 2012, p. 73-109.

29. Zimmermann M. The potential of encapsulated iron compounds in food fortification: a review. Int J Vitam Nutr Res 2004; 4(6): 453-461.

30. Zuidam N, Simoni E. Overview of microencapsulates for use in food products or processes and methods to make them. In: Zuidam, N.; Nedovic, V. (Eds.). Encapsulation Technologies for Food Active Ingredients and Food Processing. Springer. Dordrecht, Netherlands., 2010, p. 3-29.

31. Ding $B$, Zhang X, Hayat $K$, Xia S, Jia C, Xie M, Liu C. Preparation, characterization and the stability of ferrous glycinate nanoliposomes. J Food Eng 2011; 102(2): 202-208.

32. Valenzuela $C$, Hernández $V$, Morales $M$, Neira-Carrillo A, Pizarro F. Preparation and characterization of heme ironalginate beads. LWT-Food Sci Technol 2014; 59(2): 1283-1289.

33. Valenzuela C, Hernández V, Morales M, Pizarro F. Heme iron release from alginate beads at in vitro simulated gastrointestinal conditions. Biol Trace Elem Res 2016; 172(1): 251-257.

34. Perez-Moral N, Gonzalez M, Parker R. Preparation of ironloaded alginate gel beads and their release characteristics under simulated gastrointestinal conditions. Food Hydrocolloid 2013; 31(1): 114-120.

35. Al-gawhari F. Preparation of ferrous sulfate microcapsules as a sustained release dosage forms. Int I Appl Pharm 2016; 8(3): 1-4.

36. Yuan L, Geng L, Ge L, Yu P, Duan X. Effect of iron liposomes on anemia of inflammation. Int J Pharm 2013; 454: 82-89.

37. Choi S, Decker E, Mc Clements D. Impact of iron encapsulation within the interior aqueous phase of water-in-oil-in-water emulsions on lipid oxidation. Food Chem 2009; 116(1): 271-276.

38. Antileo R, Valenzuela C, Figueroa J. Characterization of a novel encapsulated oral iron supplement to prevent irondeficiency anemia in neonatal piglets. J Anim Sci doi 2016; 94: $157-160$

39. Oshinowo T, Diosady L, Yusufali R, Wesley A, Mannar V. Production of encapsulated iron for double fortification of salt with iodine and iron. https://www.researchgate.net/ publication/237604161. 2016.

40. Li Y, Diosady L, Jankowski S. Effect of iron compounds on the storage stability of multiple-fortified ultra rice ${ }^{\circledR}$. Int I Food Sci Tech 2008; 43(3): 423-429.

41. Li Y, Diosady L, Wesley A. lodine stability in iodized salt dual fortified with microencapsulated ferrous fumarate made by an extrusion-based encapsulation process. J Food Eng 2010; 99(2): 232-238.

42. Abbasi S, Azari S. Efficiency of novel iron microencapsulation techniques: fortification of milk. Int J Food Sci Tech 2011; 46(9): 1927-1933.

43. Jayalalitha V, Balasundaram B, Kumar C. Fortifiication of encapsulated iron in probiotic yoghurt. Int I Agr Res Rev 2012; 2(2): 80-84.

44. Zariwala M, Elsaid N, Jackson T, López F, Farnaud S, Somavarapu $S$, Renshaw D. A novel approach to oral iron delivery using ferrous sulphate loaded solid lipid nanoparticles. Int J Pharm 2013; 456(2): 400-407.

45. Gupta C, Chawla P, Arora S, Tomar S, Singh A. Iron microencapsulation with blend of gum arabic, maltodextrin and modified starch using modified solvent evaporation method-Milk fortification. Food Hydrocolloid 2014; 43: 622-628.

46. Zlotkin S, Arthur P, Yeboah K, Yeung, G. Treatment of anemia with microencapsulated ferrous fumarate plus ascorbic acid supplied as sprinkles to complementary (weaning) foods. Am J Clin Nutr 2001; 74: 791-795.

47. Zimmermann M, Zeder C, Chaouki N, Saad A, Torresani $T$, Hurrell R. Dual fortification of salt with iodine and microencapsulated iron: a randomized, double- blind, controlled trial in Moroccan schoolchildren. Am J Clin Nutr 2003; 77: 425-432.

48. Tondeur M, Schauer C, Christofides A, Asante K, Newton S, Serfass $R$, Zlotkin $S$. Determination of iron absorption from intrinsically labeled microencapsulated ferrous fumarate (sprinkles) in infants with different iron and hematologic status by using a dual-stable-isotope method. Am I Clin Nutr 2004; 80(5): 1436-1444.

49. Barbosa T, Taddei J, Palma, D, Ancona-Lopez F, Braga J. Double-blind randomized controlled trial of rolls fortified with microencapsulated iron. Rev Assoc Med Bras 2012; 58(1): 118-124.

50. Contreras C, Barnuevo M, Guillén I, Luque A, Lázaro E, Espadaler J, López-Román J, Villegas J. Comparative study of the oral absorption of microencapsulated ferric saccharate and ferrous sulfate in humans. Eur J Nutr 2014; 53(2): 567574.

51. Yuan L, Geng L, Geb L, Yub P, Duanb X, Chend J, Changb 
Y. Effect of iron liposomes on anemia of inflammation. Int J Appl Pharm 2013; 454(1): 82-89.

52. Xu Z, Liu S, Wang H, Gao G, Yu P, Chang Y. Encapsulation of iron in liposomes significantly improved the efficiency of iron supplementation in strenuously exercised rats. Biol Trace Elem Res 2014; 162: 181-188.

53. Zlotkin S, Antwi K, Schauer C, Yeung G. Use of microencapsulated iron (II) fumarate sprinkles to prevent recurrence of anaemia in infants and young children at high risk. B World Health
Organ 2003; 81(2): 108-115.

54. Zlotkin S, Schauer C, Christofides A, Sharieff W, Tondeur $M$, Hyder S. Micronutrient sprinkles to control childhood anaemia. PLoS Med 2005; 2(1): e1.

55. Gaucheron F. Iron fortification in dairy industry. Trends Food Sci Tech 2000; 11: 403-409.

56. Martınez-Navarrete N, Camacho M. M, Martınez-Lahuerta J, Martınez-Monzó J, Fito P. Iron deficiency and iron fortified foods-a review. Food Res Int 2002; 35(2): 225-231. 